\title{
TRES TEOREMAS SOBRE CARDINALES MEDIBLES
}

\author{
FRANKLIN GALINDO
}

\begin{abstract}
RESUMEN. El estudio de los «cardinales grandes» es uno de los principales temas de investigación de la teoría de conjuntos y de la teoría de modelos que ha contribuido con el desarrollo de dichas disciplinas. Existe una gran variedad de tales cardinales, por ejemplo cardinales inaccesibles, débilmente compactos, Ramsey, medibles, supercompactos, etc. Tres valiosos teoremas clásicos sobre cardinales medibles son los siguientes: (i) compacidad débil, (ii) Si $\kappa$ es un cardinal medible, entonces $\kappa$ es un cardinal inaccesible $y$ existen $\kappa$ cardinales inaccesibles menores que $\kappa$, y (iii) Si existe un cardinal medible, entonces el axioma de constructibilidad $(V=L)$ es falso. El objetivo de este artículo es presentar una demostración de cada uno de estos tres teoremas en el contexto de la teoría de modelos usando ideas del texto [3]. Tales demostraciones tienen en común el uso del método de construcción de modelos llamado ultraproductos, de lógicas infinitarias o fragmentos de la lógica de segundo orden y del axioma de elección. Cardinales grandes y/o ultraproductos son importantes en teoría de conjuntos, teoría de modelos, análisis matemático, teoría de la medida, probabilidades, topología, análisis funcional, física, teoría de números, finanzas, etc.
\end{abstract}

\section{INTRODUCCIÓN}

El estudio de los «cardinales grandes» es uno de los principales temas de investigación de la teoría de conjuntos y de la teoría de modelos que ha contribuido con el desarrollo de dichas disciplinas. Existe una gran variedad de tales cardinales, por ejemplo cardinales inaccesibles, débilmente compactos, Ramsey, medibles, supercompactos, etc. Tres valiosos teoremas clásicos sobre cadinales medibles son los siguientes (ver $[3,6,16]$ ): (i) compacidad débil, (ii) Si $\kappa$ es un cardinal medible, entonces $\kappa$ es un cardinal inaccesible y existen $\kappa$ cardinales inaccesibles menores que $\kappa$, y (iii) Si existe un cardinal medible, entonces el axioma de constructibilidad ( $V=L$ ) es falso. El objetivo de este artículo es presentar una demostración de cada uno de estos tres teoremas en el contexto de la teoría de modelos usando ideas del texto «Model Theory» de Chang y Keisler [3. Tales demostraciones tienen en común el uso del método de construcción de modelos llamado ultraproductos, de lógicas infinitarias o fragmentos de la lógica de segundo orden y del axioma de elección. Vale la pena resaltar que los cardinales grandes y/o los ultraproductos son importantes en teoría de conjuntos, teoría de modelos, análisis matemático, teoría de la medida, probabilidades, topología, análisis funcional, física, teoría de números, finanzas, etc. (ver $[2,3,6,13,15,16,19]$ )

Existen otras demostraciones interesantes de los teoremas que se demostrarán en este artículo que también usan ultraproductos, pero que son distintas a las que aquí se presentarán. Algunas de ellas se pueden encontrar en los siguientes textos o notas $[6,13,15,16]$.

Se ofrecerán en este artículo dos referencias de problemas abiertos sobre cardinales medibles, cardinales grandes y ultraproductos.

El orden expositivo del contenido de este artículo es el siguiente: En la siguiente sección (2) se presentarán algunos conceptos preliminares y el teorema fundamental de ultraproductos (teorema de Lós). En la sección 3 se presentará el primer teorema sobre cadinales medibles (i). En la sección 4 se presentará el segundo teorema sobre cardinales medibles (ii). Y en la última sección (5) se presentará el tercer teorema sobre

2010 Mathematics Subject Classification. 03E55, 03E10, 03C20.

Palabras clave. cardinales medibles, cardinales grandes, teorema de Scott, ultraproductos. 
cadinales medibles (iii) y al final de la sección se ofrecerán dos referencias de problemas abiertos sobre sobre cardinales medibles, cardinales grandes y ultraproductos.

\section{Conceptos PRELiminares y EL TEOREMa Fundamental DE ULTRAPRODUCTOS}

En esta sección se presentará el método de construcción de modelos llamado ultraproductos en el contexto de la teoría de modelos siguiendo principalmente el texto [3] cap.4]. Esto significa que se usará informalmente la teoría axiomática de conjuntos de Zermelo-Fraenkel con el axioma de elección (ZFC) tal como es presentada y desarrollada en los textos $[5,8,12,13,15,17]$, entre otros. Específicamente se presentará esta sección siguiendo el artículo [10] (el cual está en la web), en dicho artículo se describe a los ultraproductos, a una demostración del teorema fundametal de los ultraproductos, y a una prueba del teorema de compacidad usando ultraproductos. El método de ultraproductos se originó con Skolem en 1930 y luego fue desarrollado por Loś en 1955 (ver $[3,13,18])$.

La presentación se realizará en el siguiente orden expositivo: En las subsecciones 2.1 y 2.2 se ofrecerán algunos conceptos y resultados necesarios para definir los ultraproductos, por ejemplo «filtro», «ultrafiltro», «lema de Zorn», «teorema del ultrafiltro», etc. Y se mencionarán sin definir otros conceptos y resultados de la teoría de modelos que son necesarios para desarrollar este artículo pero que son básicos y se presupone que el lector tiene este conocimiento, por ejemplo la sintaxis y la semántica de la lógica de primer orden y algunas relaciones entre estructuras. Y en la subsección 2.3 se definirán los ultraproductos y se formulará el teorema fundamental de los ultraproductos siguiendo ideas principalmente de $[3,13,16,21]$.

\subsection{Filtros, ultrafiltros, lema de Zorn, teorema del ultrafiltro.}

Definición 1. - Un filtro sobre un conjunto no vacío $S$ es una colección $\mathcal{F}$ de subconjuntos de $S$ tal que:

(i) $S \in \mathcal{F}$ y $\emptyset \notin \mathcal{F}$.

(ii) Si $\mathcal{F} F$ y $Y \in \mathcal{F}$, entonces $X \cap Y \in \mathcal{F}$.

(iii) Si $X \in \mathcal{F}$ y $X \subseteq Y$, entonces $Y \in \mathcal{F}$.

- Sea $\mathcal{F}$ un filtro sobre un conjunto $S$. $\mathcal{F}$ es un ultrafiltro si para cualquier $X \subseteq S$ se cumple que:

$$
X \in \mathcal{F} \leftrightarrow S \backslash X \notin \mathcal{F} .
$$

- Sea $\mathcal{F}$ un ultrafiltro sobre un conjunto $S . \mathcal{F}$ es no principal si y sólo si $\forall i \in S(\{i\} \notin F)$.

Ejemplos de filtros (ver [3,13]): (1) filtro trivial: $\mathcal{F}=\{S\}$. (2) Para cada $B \subseteq S$, $B \neq \emptyset$, el filtro $\mathcal{F}_{B}=\{Z \subseteq S: B \subseteq Z\}$ se llama filtro principal generado por $B$. Para $B=\{a\} \subseteq S, \mathcal{F}_{B}$ es escribe $\mathcal{F}_{a}, \mathcal{F}_{a}=\{Z \subseteq S: a \in Z\}$. Nótese que $\mathcal{F}_{a}$ es un ultrafiltro principal. (3) Sea $S$ un conjunto infinito, el filtro $\mathcal{F}=\left\{X \in P(S):|S \backslash X|<\aleph_{0}\right\}$ se llama filtro de Fréchet. Nótese que el filtro de Fréchet no es principal. Dado cualquier conjunto infinito $S$ siempre se puede construir un filtro no principal sobre $S$, que extiende al filtro de Fréchet sobre $S$ usando la propiedad de intersección finita, tal como lo afirma el teorema que viene a continuación (Teorema 2). Pregunta: Ya se demostró anteriormente que existen ultrafiltros principales, mediante un ejemplo, $\mathcal{F}_{a}$. Pero, ¿Existen ultrafiltros no principales?. La existencia de tales entidades matemáticas sólo se puede garantizar usando el lema de Zorn, no hay otra manera (ver [13], p. 75]). A continuación se presentará (después del Teorema 2) el teorema del ultrafiltro, el cual permitirá contar con tales entidades (ultrafiltros no principales) las cuales son fundamentales para la investigación matemática, por ejemplo para la construcción del cuerpo ordenado y no arquimediano de los hiper-reales del análisis no estándar de Robinson [2], y jugarán un importante papel en la definición de los cardinales medibles. 
El teorema del ultrafiltro se prueba a partir del lema de Zorn, lema que se formulará también en este trabajo.

Una familia $G$ de conjuntos tiene la propiedad de intersección finita si para cualquier conjunto finito $H=\left\{X_{1}, \ldots, X_{n}\right\} \subseteq G$ se cumple que $H_{1} \cap \ldots \cap H_{n} \neq \emptyset$.

Teorema 2. 1. Si $\Delta$ es una familia de filtros sobre $S$, entonces $\bigcap \Delta$ es un filtro sobre $S$.

2. Si $\Omega$ es una $\subseteq$-cadena de filtros sobre $S$ (es decir, $\forall X, Y \in \Omega(X \subseteq Y o Y \subseteq X)$ ), entonces $\bigcup \Omega$ es un filtro sobre $S$.

3. Si $G \subseteq P(S)$ tiene la propiedad de intersección finita, entonces existe un filtro $\mathcal{F}$ tal $G \subseteq \mathcal{F}\left(\mathcal{F}=\left\{X \subseteq S: \exists Z_{1}, \ldots, Z_{n} \in G\left(Z_{1} \cap \ldots \cap Z_{n} \subseteq X\right)\right\}\right)$.

Una prueba de este resultado puede encontrarse (entre otros) en [[13, p.74] y [ [3], p. 212].

Teorema 3 (Teorema del ultrafiltro(Tarski)). Todo filtro se puede extender a un ultrafiltro.

Como ya se dijo antes la demostración del teorema del ultrafiltro requiere del lema de Zorn, una versión de dicha prueba puede encontrarse (entre otros) en [13], p. 75] y [[3], p. 214]. A continuación se enuncia el lema de Zorn después de presentar unas definiciones previas:

Definición 4. Sea $\mathrm{A}$ un conjunto y $\mathrm{R}$ una relación binaria en $\mathrm{A}$ (es decir, $R \subseteq A \times A$ )

1. $\mathrm{R}$ es reflexiva si y sólo si $\forall x \in A(x R x)$

2. $\mathrm{R}$ es simétrica si y sólo si $\forall x, y \in A(x R y \rightarrow y R x)$

3. $\mathrm{R}$ es transitiva si y sólo si $\forall x, y, z \in A(x R y \wedge y R z \rightarrow x R z)$

4. $\mathrm{R}$ es antisimétrica si y sólo si $\forall x, y \in A(x R y \wedge y R x \rightarrow x=y)$.

5. $\mathrm{R}$ es una relación de equivalencia si $\mathrm{R}$ es una relación reflexiva, simétria $\mathrm{y}$ transitiva.

Definición 5. 1. Un orden parcial es un par $(P, \leq)$ donde $P$ es un conjunto no vacío y $\leq$ es una relación en $P$ que es reflexiva, antisimétrica y transitiva.

2. Dado un orden parcial $(P, \leq)$ se dice que $p<q \leftrightarrow p \leq q \wedge p \neq q$.

3. Sean $(P, R)$ un orden parcial y $D \subseteq P . x \in P$ es un elemento mínimo (máximo) de $\mathrm{D}$ si $x \in D \wedge$ no existe ningún $y \in D$ tal que $y \neq x \wedge y R x(x R y)$. $\mathrm{x}$ es una cota inferior (superior) de D si $\forall y \in D(x R y \vee y=x)(y R x \vee y=x)$. $\mathrm{x}$ es un infimo (supremo) de $\mathrm{D}$ si $\mathrm{x}$ es cota inferior (superior) de $\mathrm{D} \wedge$ para todo $y \in P$, si y es una cota inferior (superior) de $\mathrm{D}$, entonces $y R x \vee y=x(x R y \vee y=x)$. $\mathrm{x}$ es un menor (mayor) elemento de D si $x \in D \wedge \forall y \in D(x R y \vee y=x)(y R x \vee y=$ $x)$.

4. Sea $(P, R)$ un orden parcial. $(P, R)$ es un orden total (o lineal) si la relación $\mathrm{R}$ satisface la propiedad de tricotomía: $\forall x, y \in P(x R y \vee y R x \vee x=y)$.

5. Sea $(P, R)$ un orden parcial. $(P, R)$ es un buen orden si para todo $X \subseteq P$ se cumple que: Si $X \neq \emptyset$, entonces $X$ tiene un menor elemento. (Nótese que todo conjunto bien ordenado es un conjunto totalmente ordenado). $P)$

(Un orden parcial u orden total o buen orden $(P, \leq)$ a veces se denotará por

Lema 6 (Lema de Zorn). Sea $(K, S)$ un conjunto parcialmente ordenado tal que cada $X \subseteq K$ totalmente ordenado tiene una cota superior en $K$. Entonces $K$ tiene un elemento máximo.

Es conocido que el lema de Zorn es equivalente al axioma de elección. Donde el axioma de elección es la siguiente sentencia: Todo conjunto tiene una función selectora. (Dado un conjunto $Z$ se dice que la función $f$ es una función de elección - o una función selectora - para $Z$ si el $\operatorname{dom}(f)=Z-\{\emptyset\}$ y para todo $W \in \operatorname{dom}(f)$, se tiene que $f(W) \in W)$. Una prueba de la equivalencia puede encontrarse (entre otros) en [[5], 
p. 83-85] y [ [8], p. 151-153]. También es conocido que el lema de Zorn es equivalente al principio del buen orden (Todo conjunto se puede bien ordenar). Una prueba de tal equivalencia puede encontrarse (entre otros) en [[5], p. 82-85] y [8], p. 151-153, 196-197].

2.2. Conceptos básicos de la teoría de modelos: sintaxis y semántica de la lógica de primer orden, relaciones entre estructuras. Los conceptos básicos de la teoría de modelos, como por ejemplo la sintaxis y la semántica de la lógica de primer orden y las relaciones básicas entre entructuras, que se usarán en este artículo se suponen conocidas por parte del lector, no se describirán, son las mismas nociones que están formuladas y explicadas en los textos contemporáneos de lógica matemática o de teoría de modelos, por ejemplo en $[3,4,7,9,19,21]$. Un resumen de tales conceptos puede conseguirse en [10, entre otros. Tales nociones son: «lenguajes de primer orden», «estructuras», «formalización de un lenguaje (en primer orden)», «fórmula», «sentencia», «satisfacción y verdad en una estructura», «modelo», «contradicción», «consecuencia lógica», relaciones entre estructuras: «isomorfismo», «subestructura», «inmersión», «submodelo elemental», «inmersión elemental», «equivalencia elemental», etc.

2.3. El teorema fundamental de ultraproductos. ¿Cómo se contruyen las estructuras (modelos) llamadas ultraproductos? La construcción se realiza usando (entre otros) a los ultrafiltros, en este artículo se presenta tal construcción siguiendo principalmente los textos [[3, cap. 4], [[21, p. 129-135] y [[13, p. 158-161].

Supongamos que $I$ es un subconjunto no vacío, $D$ es un filtro sobre $I$ y que para cada $i \in I, A_{i}$ es un subconjunto no vacío. Entonces se considera el producto cartesiano de los conjuntos $A_{i}$, es decir:

$$
C=\prod_{i \in I} A_{i}=\left\{f: f: I \longrightarrow \cup_{i \in I} A_{i} \wedge \forall i \in I\left(f(i) \in A_{i}\right)\right\} .
$$

Ahora se define una relación de equivalencia $\sim$ en $\prod_{i \in I} A_{i}$ de la siguiente manera:

$$
f \sim g \longleftrightarrow\{i \in I: f(i)=g(i)\} \in D .
$$

Se cumple que la relación $\sim$ es reflexiva, simétrica y transitiva, por lo tanto $\sim$ es una relación de equivalencia. Entonces se considera el conjunto cociente de $\prod_{i \in I} A_{i}$ determinado por $\sim$ (llamado producto reducido de los $A_{i}$ modulo $\left.D\right)$ :

$$
\prod_{i \in I} A_{i} / \sim=\left\{[f]: f \in \prod_{i \in I} A_{i}\right\}
$$

Se denotará al conjunto cociente $\prod_{i \in I} A_{i} / \sim$ por $\prod_{D} A_{i}$ y la clase de equivalencia $[f]$ se denotará por $f_{D} \quad\left(\forall f \in \prod_{i \in I} A_{i}\right)$. Cuando $D$ es un ultrafiltro $\prod_{D} A_{i}$ es llamado un ultraproducto. $\mathrm{Y}$ en el caso de que todos los conjuntos $A_{i}$ sean iguales, digamos, $A_{i}=A(\forall i \in I), \prod_{D} A$ es llamada la ultrapotencia de $A$ modulo $D$.

Ahora se definirá el producto reducido de modelos para un lenguaje $\mathcal{L}$ fijo:

$\left(\mathcal{L}\right.$ es un conjunto, de cualquier cardinalidad, de símbolos de relación $\left(R_{0}, R_{1}\right.$, $\ldots)$, símbolos de función $\left(F_{0}, F_{1}, \ldots\right)$ y de símbolos constantes $\left(c_{0}, c_{1}, \ldots\right)$. Donde el subconjunto de $\mathcal{L}$ de símbolos de relación puede ser vacío, el subconjunto de $\mathcal{L}$ de símbolos de función puede ser vacío, y el subconjunto de $\mathcal{L}$ de símbolos constantes puede ser vacío. Cada símbolo de relación y cada símbolo de función tiene asociado un número natural $\geq 1$, su número de argumentos, de modo que tenemos símbolos de relación o de función unarios, binarios, ..., n-arios, etc.)

Sea $I$ un conjunto no vacío, $D$ un filtro sobre $I$ y para cada $i \in I$ sea $\mathfrak{A}_{i}$ una estructura para un lenguaje $\mathcal{L}$. Supongamos que para cada símbolo relacional $P$ de $\mathcal{L}$ la interpretación de $P$ en $\mathfrak{A}_{i}$ es $R_{i}$, los símbolos de función $F$ son interpretados en $\mathfrak{A}_{i}$ por $G_{i}$ y los símbolos constantes $c$ son interpretados en $\mathfrak{A}_{i}$ por $a_{i}$. 
El producto reducido $\prod_{D} \boldsymbol{A}_{i}$ es una estructura para $\mathcal{L}$ definida de la siguiente manera:

(i) El universo de $\prod_{D} \boldsymbol{\mathfrak { A }}_{i}$ es $\prod_{D} A_{i}$.

(ii) Sea $P$ un símbolo de relación $n$-ario de $\mathcal{L}$. La interpretación de $P$ en $\prod_{D} \mathfrak{A}_{i}$ es la relación n-ária $S$ definida de la siguiente manera:

$$
S\left(f_{D}^{1}, \ldots, f_{D}^{n}\right) \longleftrightarrow\left\{i \in I: R_{i}\left(f^{1}(i), \ldots, f^{n}(i)\right)\right\} \in D .
$$

(iii) Sea $F$ un símbolo de función $n$-ario de $\mathcal{L}$. Entonces $F$ es interpretado en $\prod_{D} \mathfrak{A}_{i}$ por la función n-ária $H$ definida de la siguiente manera:

$$
H\left(f_{D}^{1}, \ldots, f_{D}^{n}\right)=\left\langle G_{i}\left(f^{1}(i), \ldots, f^{n}(i)\right): i \in I\right\rangle_{D}
$$

(iv) Sea $c$ una constanta de $\mathcal{L}$. Entonces la interpretación de $c$ en $\prod_{D} \mathfrak{A}_{i}$ es un $b \in \prod_{D} \mathfrak{A}_{i}$ que se define como sigue:

$$
b=\left\langle a_{i}: i \in I\right\rangle_{D} .
$$

Se cumple que las definiciones realizadas anteriormente de $S\left(f_{D}^{1}, \ldots, f_{D}^{n}\right)$ y $H\left(f_{D}^{1}, \ldots, f_{D}^{n}\right)$ dependen solamente de las clases de equivalencia y no de sus representantes $f^{1}, \ldots, f^{n}$. Es decir, $f^{1} \sim g^{1}, \ldots, f^{n} \sim g^{n}$, entonces :

$$
\left\{i \in I: R_{i}\left(f^{1}(i), \ldots, f^{n}(i)\right)\right\} \in D \leftrightarrow\left\{i \in I: R_{i}\left(g^{1}(i), \ldots, g^{n}(i)\right)\right\} \in D
$$

y

$$
\left\langle G_{i}\left(f^{1}(i), \ldots, f^{n}(i)\right): i \in I\right\rangle_{D}=\left\langle G_{i}\left(g^{1}(i), \ldots, g^{n}(i)\right): i \in I\right\rangle_{D} .
$$

Ahora de formulará el teorema fundamental de ultraproductos (Loś, 1955, [Lo]) y dos corolarios del mismo:

Teorema 7 (Teorema fundamental de ultraproductos (Loś)). Sea $\mathfrak{B}$ el ultraproducto $\prod_{D} \mathfrak{A}_{i}$, donde $I$ es el conjunto de índices de los $\mathfrak{A}_{i}$. Entonces :

(i) Para cada término $t\left(x_{1}, \ldots, x_{n}\right)$ de $\mathcal{L}$ y elementos $f_{D}^{1}, \ldots, f_{D}^{n} \in B=\prod_{D} A_{i}$ se tiene que:

$$
t_{\mathfrak{B}}\left[f_{D}^{1}, \ldots, f_{D}^{n}\right]=\left\langle t_{\mathfrak{A}_{i}}\left[f^{1}(i), \ldots, f^{n}(i)\right]: i \in I\right\rangle_{D}
$$

(ii) Dada una fórmula $\varphi\left(x_{1}, \ldots, x_{n}\right)$ de $\mathcal{L}$ y $f_{D}^{1}, \ldots, f_{D}^{n} \in B$ se tiene que:

$$
\mathfrak{B} \models \varphi\left[f_{D}^{1}, \ldots, f_{D}^{n}\right] \leftrightarrow\left\{i \in I: \mathfrak{A}_{i} \models \varphi\left[f^{1}(i), \ldots, f^{n}(i)\right]\right\} \in D
$$

(iii) Para cada sentencia $\varphi$ de $\mathcal{L}$ se tiene que:

$$
\mathfrak{B} \models \varphi \leftrightarrow\left\{i \in I: \mathfrak{A}_{i}=\varphi\right\} \in D .
$$

(Inuitivamente (iii) afirma que $\varphi$ es verdadera en el ultraproducto $\mathfrak{B}=\prod_{D} \mathfrak{A}_{i}$ si y sólo si $\varphi$ es verdadera en "casi todos" los factores $\mathfrak{\mathfrak { A }}_{i}$ de $\mathfrak{B}$ )

Una prueba de este teorema puede encontrarse en $[3,10,13,21]$, entre otros.

Corolario 8. Sea $\mathfrak{A}$ una estructura para un lenguaje $\mathcal{L}$ y sea $\prod_{D} \mathfrak{A}$ una ultrapotencia de $\mathfrak{A}$. Entonces $\mathfrak{A} \equiv \prod_{D} \mathfrak{A}$.

Una prueba de este corolario puede encontrarse en [21, entre otros.

Para enunciar el segundo corolario se requiere de una difinición previa: Sea $I$ un conjunto no vacío, $D$ un filtro sobre $I$ y $\mathfrak{A}$ una estructura para un lenguaje $\mathcal{L}$. La inmersión natural de $\mathfrak{A}$ dentro de $\prod_{D} \mathfrak{A}$ es la función $d: \mathfrak{A} \longrightarrow \prod_{D} \mathfrak{A}$ definda com sigue: $\forall a \in A\left(d(a)=\langle a: i \in I\rangle_{D}\right)$. El rango de $d$ es denotado por $d(A)$ y la restricción de $\prod_{D} \mathfrak{A}$ a $d(A)$ es donotada por $d(\mathfrak{A})$.

Corolario 9. Sea $\mathfrak{A}$ una estructura para un lenguaje $\mathcal{L}$ y $D$ un ultrafiltro. Entonces la inmersión natural de $\mathfrak{A}$ dentro de la ultrapotencia $\prod_{D} \mathfrak{A}$ es una inmersión elemental. 
Una prueba de este corolario puede encontrarse en $[3,10,13,21]$, entre otros.

\section{PRIMER TEOREMA SOBRE CARDINALES MEDIBLES: COMPACIDAD DÉBIL}

Se iniciará esta sección presentando la definición de «cofinalidad», «cardinal regular», «cardinal inaccesible», «cardinal medible»y «lógicas infintarias», las cuales son necesarias para desarrollar la misma.

Sea $\alpha$ un ordinal límite. Decimos que $\beta<\alpha$ es cofinal con $\alpha$ si existe una función creciente $f: \beta \longrightarrow \alpha$ tal que para todo $\xi<\alpha$, existe un $\delta<\beta$ tal $f(\delta) \geq \xi$ (es decir, la imagen de $f$ es no acotada en $\alpha$ ). Dado $\alpha$, la cofinalidad de $\alpha, \operatorname{cof}(\alpha)$, es el menor ordinal cofinal $\operatorname{con} \alpha$. Con respecto a la cofinalidad se cumple lo siguiente: $\operatorname{cof}(\alpha)$ es el menor cardinal $\beta$ tal que existe una partición de $\alpha$ en $\beta$ pedazos cada uno de los cuales tiene cardinalidad estrictamente menor que $\alpha$. Un cardinal infinito $\kappa$ es regular si es igual a su cofinalidad. Decimos que es singular en caso contrario $(\operatorname{cof}(\alpha)<\alpha)$. Un cardinal $\kappa$ es un cardinal limite fuerte si para todo cardinal $\theta<\kappa$ se tiene que $2^{\theta}<\kappa$. Un cardinal $\kappa>\aleph_{0}$ es inaccesible si es regular y límite fuerte (Nótese que si se quita la condición de que $\kappa>\aleph_{0}$ se tiene que $\aleph_{0}$ es un cardinal inaccesible).

Sea $\alpha$ un cadinal infinito. Un filtro $D$ sobre $I$ se llama $\alpha$-completo si y sólo si: $X \subseteq D$ y $|X|<\alpha$ implica $\bigcap X \in D$. Un cardinal $\alpha>\aleph_{0}$ se dice que es medible si y sólo si existe un ultrafiltro no principal y $\alpha$-completo sobre $\alpha$ (Nótese que si se quita la condición de que $\kappa>\aleph_{0}$ se tiene que $\aleph_{0}$ es un cardinal medible).

Se definen ahora los lenguajes infinitarios $\mathcal{L}_{\kappa \kappa}$, donde $\kappa$ es un cardinal mayor o igual que $\aleph_{0}$, siguiendo a $[1,3,5,9]$ :

Considérese un lenguaje de primer orden $\mathcal{L}$ (conjunto de símbolos relacionales, funcionales y constantes) y también considérese las reglas que se usan para formalizar el mismo, es decir, para construir las fórmulas del lenguaje $\mathcal{L}$. A la lista de símbolos lógicos agréguese los siguientes nuevos símbolos: $\bigwedge$ (conjuntor infinito) y $\bigvee$ (disyuntor infinito), y sustitúyase la lista numerable de variables (que se usa normalmente) por una lista de variables de cardinal $\kappa\left(V A R_{\mathcal{L}_{\kappa \kappa}}\right)$. Para construir el conjunto de fórmulas de $\mathcal{L}_{\kappa \kappa}$ se utilizan las mimas reglas que se usan para contruir las fórmulas de lenguaje $\mathcal{L}$ (ver alguna definición usual), más las siguientes dos nuevas reglas:

$(\odot 1)$ Si $\Phi$ es un conjunto de fórmulas de $\mathcal{L}_{\kappa \kappa}$ tal que $|\Phi|<\kappa$, entonces $\bigwedge \Phi$ y $\bigvee \Phi$ también son formulas de $\mathcal{L}_{\kappa \kappa}$.

$(\odot 2) \operatorname{Si} \varphi$ es una fórmula de $\mathcal{L}_{\kappa \kappa}$ y $V$ es un conjunto de variables tal que $|V|<\kappa$, entonces $(\forall V) \Phi$ y $(\exists V) \Phi$ también son fórmulas de $\mathcal{L}_{\kappa \kappa}$.

Semántica de $\mathcal{L}_{\kappa \kappa}$ : Una estructura para evaluar las formulas del lenguaje $\mathcal{L}_{\kappa \kappa}$ es igual que una estructura para el lenguaje $\mathcal{L}$, pues tiene los mismos símbolos no lógicos. Sea $\phi$ una fórmula de $\mathcal{L}_{\kappa \kappa}, \mathfrak{C}$ una estructura para $\mathcal{L}$ y $s: V A R_{\mathcal{L}_{\kappa \kappa}} \longrightarrow C$. La definición de $\mathfrak{C}=\phi[s]$ ( $s$ satisface a $\phi$ en $\mathfrak{C}$ ) es igual que la definición usual que se hace para $\mathcal{L}$, más las siguientes reglas correspondientes a los nuevas fórmulas construídas mediante $(\odot 1)$ y $(\odot 2)$ :

$(\odot 1.1) \mathfrak{C} \models \bigwedge \Phi[s] \Longleftrightarrow \mathfrak{C}=\varphi[s]$, para toda $\varphi \in \Phi$.

$(\odot 1.2) \mathfrak{C}=\bigvee \Phi[s] \Longleftrightarrow \mathfrak{C}=\varphi[s]$, para alguna $\varphi \in \Phi$.

$(\odot 2.1) \mathfrak{C}=((\forall V) \chi)[s] \Longleftrightarrow \mathfrak{C} \models \chi\left[s^{\prime}\right]$ para toda $s^{\prime}: V A R_{\mathcal{L}_{\kappa \kappa}} \longrightarrow C$ que difiere de $s$ a lo sumo en los valores de las variables de $V$.

$(\odot 2.2) \mathfrak{C}=((\exists V) \chi)[s] \Longleftrightarrow \mathfrak{C}=\chi\left[s^{\prime}\right]$ para alguna $s^{\prime}: V A R_{\mathcal{L}_{\kappa \kappa}} \longrightarrow C$ que difiere de $s$ a lo sumo en los valores de las variables de $V$.

Nótese que si $\kappa=\aleph_{0}$, entonces $\mathcal{L}_{\aleph_{0} \aleph_{0}}$ es la lógica de primer orden usual.

Es importante destacar, porque se utilizará más adelante, que con dicho lenguaje infinitario, en particular con $\kappa>\aleph_{0}$ se puede caracterizar el concepto de «relación bien fundamentada» (una relación binaria $\mathrm{R}$ es bien fundamentada si no existen cadenas infinitas descendientes con respecto a $\mathrm{R}$, por ejemplo «€» en $\mathbf{V}$ ) y el concepto de «relación bien ordenada». En efecto: La siguiente sentencia afirma que la relación binaria que nombra el símbolo relacional binario $P(x, y)$ es bien fundamentada: 


$$
\mathrm{RBF}:\left(\forall x_{0} x_{1} x_{2} \ldots\right) \neg \bigwedge\left\{P\left(x_{n+1}, x_{n}\right): n \in \aleph_{0}\right\},
$$

y cuando se adiciona (con la conjunción finita $\wedge$ ) a la sentencia anterior los axiomas de orden total escritos con el lenguaje $\mathcal{L}$,

$$
\text { RBO: }\left[\left(\forall x_{0} x_{1} x_{2} \ldots\right) \neg \bigwedge\left\{P\left(x_{n+1}, x_{n}\right): n \in \aleph_{0}\right\}\right] \wedge \text { Axiomas de orden total, }
$$

se tiene que la relación determinada por $P(x, y)$ es un buen orden en la estructura respectiva donde $P(x, y)$ sea interpretado y se satisfaga la sentencia RBO.

El teorema fundamental de ultraproductos tiene versiones más fuertes. En efecto, se cumple que los ultraproductos también preservan las fórmulas infinitarias (si se le agrega una hipótesis adicional al ultrafiltro, $\delta$-completo, $\delta$ un cardinal mayor o igual que $\aleph_{0}$ ), ese es el significado del siguiente resultado, una prueba del mismo puede encontrarse en [[3], p. 231-232]:

LEMa 10 (Los ultraproductos también preservan las fórmulas infinitarias). Sea $\mathfrak{B}$ el ultraproducto $\prod_{D} \mathfrak{A}_{i}$, donde $I$ es el conjunto de índices de los $\mathfrak{A}_{i}$, y $D$ es un ultrafiltro $\delta$-completo ( $\delta$ un cardinal mayor o igual que $\aleph_{0}$ ). Entonces:

(i) Dada una fórmula $\varphi\left(x_{1}, \ldots, x_{n}, x_{n+1}, \ldots\right)$ de $\mathcal{L}_{\delta \delta}$ y $f_{D}^{1}, \ldots, f_{D}^{n}, f_{D}^{n+1}, \ldots \in B$ se tiene que:

$$
\begin{gathered}
\mathfrak{B} \models \varphi\left[f_{D}^{1}, \ldots, f_{D}^{n}, f_{D}^{n+1}, \ldots\right] \leftrightarrow \\
\left\{i \in I: \mathfrak{A}_{i} \models \varphi\left[f^{1}(i), \ldots, f^{n}(i), f^{n+1}(i), \ldots\right]\right\} \in D
\end{gathered}
$$

(ii) Para cada sentencia $\varphi$ de $\mathcal{L}_{\delta \delta}$ se tiene que:

$$
\mathfrak{B} \models \varphi \leftrightarrow\left\{i \in I: \mathfrak{A}_{i}=\varphi\right\} \in D
$$

El siguiente teorema es una versión del teorema de compacidad (de la lógica de primer orden) para conjuntos de sentencias de lenguajes infinitarios cuyo cardinal sea un cardinal medible. La demostración supone ultraproductos y lenguajes infinitarios (preservados por ultraproductos). Con dicho teorema se abre la puerta para la definición de un nuevo tipo de cardinal grande: «cardinal débilmente compacto». Se cumple que «cardinal medible» implica «cardinal débilmente compacto» (y algo más fuerte), pero «cardinal débilmente compacto» no implica «cardinal medible» (ver [[3], p. 243] y [5], p. 132]). Es decir, la hipótesis «existe un cardinal medible» es más fuerte que la hipótesis «existe un cardinal débilmente compacto». Los cardinales débilmente compactos se pueden caracterizar también con propiedades de combinatoria infinita tipo Ramsey (ver [[5], p. 118]), después de la demostración del teorema se comentará brevemente este asunto.

Teorema 11 (Teorema de compacidad débil). Sea $\eta$ un cardinal medible, y $\Gamma$ un conjunto de sentencias de $\mathcal{L}_{\eta \eta}$ tal que $|\Gamma|=\eta$ y cualquier subconjunto $\Gamma_{0} \subseteq \Gamma$ tal que $\left|\Gamma_{0}\right|<\eta$ tiene un modelo. Entonces $\Gamma$ tiene un modelo.

Demostración. Como $\eta$ es medible se tiene que existe un ultrafiltro $H$ no principal y $\eta$-completo sobre $\eta$. Como $H$ no contiene conjuntos unitarios y es $\eta$-completo, entonces $H$ no contiene conjuntos de cardinal menor que $\eta$ (para ver la intuición de este hecho se puede pensar que $\eta=\aleph_{0}$, aunque obviamente si $\eta$ es medible es mayor que $\aleph_{0}$ por definición, es una sólo una idea para la intuición, y luego considerar que $H$ es el filtro de Fréchet sobre $\aleph_{0}$, de este modo el hecho descrito se aprecia claramente. Una demostración mas general y rigurosa es la siguiente: Sea $A \in H$ tal que $|A|<\eta$. Para todo $x \in A$ se cumple que $\{x\} \notin H$ porque $H$ es ultrafiltro no principal y entonces por definición no contiene conjuntos unitarios. Entonces para todo $x \in A(A \backslash\{x\} \in H)$ ya que $H$ es ultrafiltro. En consecuencia, como $H$ es $\eta$-completo se cumple que: 
$\bigcap_{x \in A}(A \backslash\{x\}) \in H$. Por lo tanto: $\emptyset=\left(\bigcap_{x \in A} A \backslash\{x\}\right) \cap A \in H$, pues $H$ es un filtro. Contradicción). Por lo tanto para cada $\delta<\eta$,

$$
\{\theta: \delta<\theta<\eta\} \in H,
$$

ya que el complemento de $\{\theta: \delta<\theta<\eta\}$ no pertenece a $H$ porque tiene cardinal menor que $\eta$, y entonces como $H$ es un ultrafiltro $\{\theta: \delta<\theta<\eta\} \in H$.

Sea la siguiente enumeración de $\Gamma: \Gamma=\left\{\phi_{\beta}: \beta<\eta\right\}$. Por hipótesis, para cada $\beta<\eta$, existe un modelo $\mathfrak{C}_{\beta}$ del conjunto $\left\{\phi_{\delta}: \delta<\beta\right\}$. Sea

el ultraproducto $\mathfrak{D}=\prod_{H} \mathfrak{C}_{\beta}$. Entonces para cada $\phi_{\delta} \in \Gamma$, se tiene que,

$$
\left\{\theta<\eta: \mathfrak{C}_{\theta}=\phi_{\delta}\right\} \supseteq\{\theta: \delta<\theta<\eta\} \in H .
$$

En consecuencia, como los ultraproductos preservan las formulas infinitarias (por el Lema 10), se cumple que el ultraproducto $\mathfrak{D}$ es un modelo de $\Gamma$. Lo que se quería demostrar.

Definición 12. Un cardinal $\kappa>\aleph_{0}$ es débilmente compacto si para todo conjunto $\Gamma$ de sentencias de $\mathcal{L}_{\kappa \kappa}$ tal que $|\Gamma|=\kappa$ ocurre lo siguiente: Si cada subconjunto de $\Gamma$ de cardinalidad menor que $\kappa$ tiene un modelo, entonces $\Gamma$ tiene un modelo.

Es decir, $\kappa$ es débilmente compacto si cumple con el teorema anteriormente demostrado, en otras palabras, un cardinal medible es débilmente compacto.

Nótese que como la lógica de primer orden $\mathcal{L}_{\aleph_{0} \aleph_{0}}$ satisface el teorema de compacidad esto implica que $\aleph_{0}$ es débilmente compacto, si se elimina la restricción de que el cardinal débilmente compacto debe ser mayor que $\aleph_{0}$, es decir, la definición de cardinal débilmente compacto es una generalización de una propiedad de $\aleph_{0}$ para cardinales no numerables, algo análogo ocurre con la noción de cardinal inaccesible y la de cardinal medible.

Como se dijo anteriormente todo cardinal medible es débilmente compacto, pero lo inverso no ocurre, débilmente compacto no implica medible, una demostración de ello puede encontrarse en [[3], p. 243].

La relación combinatoria $\kappa \rightarrow \kappa_{2}^{2}$ significa que para toda partición en dos clases del conjunto de subconjuntos de dos elementos de $\kappa$ existe un subconjunto $H \subseteq \kappa$ cuyos subconjuntos de dos elementos están todos en el misma clase y $H$ tiene cardinal $\kappa$. Esta definición se puede reexpresar de la siguiente manera teniendo presente que $[A]^{2}=\{\{x, y\}: x \in A \wedge y \in A\}$ y que $F^{\prime \prime}[A]=\{F(x): x \in A\}$. Entonces $\kappa \rightarrow \kappa_{2}^{2}$ significa que para toda función $F:[\kappa]^{2} \longrightarrow\{0,1\}$ existe un subconjunto $H \subseteq \kappa$ tal que $|H|=\kappa$ y existe un $i \in\{0,1\}$ tal que $F^{\prime \prime}[H]^{2}=\{i\}$.

Se cumple el siguiente teorema (ver [5], p. 118]):

Si $\kappa$ es inaccesible, entonces $\kappa$ es débilmente compacto si y sólo si $\kappa \rightarrow \kappa_{2}^{2}$.

Para culminar con los cardinales débilmente compactos vale la pena agregar lo siguente: cardinal débilmente compacto implica estrictamente cardinal inaccesible, es decir, la hipótesis «Existe un cardinal débilmente compacto» es más fuerte estrictamente que la hipótesis «Existe un cardinal inaccecible» (ver [[5], p. 132]).

\section{Segundo teorema sobre Cardinales medibles: «CARdinal Medible» ES MÁS FUERTE ESTRICTAMENTE QUE «CARDINAL INACCESIBLE».}

El segundo ejemplo de aplicación del método de ultraproductos en la teoría de conjuntos con cardinales medibles es la demostración del teorema: Si $\alpha$ es un cardinal medible, entonces $\alpha$ es un cardinal inaccesible y además $\alpha$ es el $\alpha$-ésimo cardinal inaccesible, es decir, existen $\alpha$ cardinales inaccesibles menores que $\alpha$. Esto significa (entre otros) que la hipótesis conjuntista «Existen cardinales medibles» es estrictamente más fuerte que la hipótesis «existen cardinales inaccesibles» (ver [3,5]). Es conocido que la existencia de cardinales inaccesibles no se puede demostrar de los axiomas estándar de la teoría de conjuntos, por el Segundo teorema de incompletitud de Gödel(1931) 
(ver $[3,5,11,13,17]$ ). De modo que es claro que tampoco se puede demostrar de los axiomas estándar de la teoría de conjuntos la existencia de los cardinales medibles ni la existencia de ningún otro cardinal más fuerte que inaccesible.

Vale la pena resaltar que en la prueba del teorema de esta sección se usarán lógicas infinitarias (como en el teorema anterior) y lógicas de segundo orden de un tipo específico, $\Sigma_{1}^{1}$ fórmulas, las cuales son preservadas por los ultraproductos. Algunos resultados y definiciones que se presuponen en la prueba se enuncian a continuación:

El siguiente resultado se refiere a la expansión de productos reducidos, una prueba del mismo puede encontrarse en [3], p. 216-217]:

Lema 13 (Teorema de expansión de productos reducidos). Sea un lenguaje $\mathcal{L}^{\prime}$ que expande a un lenguaje $\mathcal{L}$. Sea $I$ un conjunto no vacío y para cada $i \in I$ sea $\mathfrak{A}_{i}$ una estructura para $\mathcal{L}$, y $\mathfrak{B}_{i}$ una expansión de $\mathfrak{A}_{i}$ para $\mathcal{L}^{\prime}$ (es decir, $\mathfrak{B}_{i}$ restringida a $\mathcal{L}$ es $\mathfrak{A}_{i}$, en otras palabras, los universos de $\mathfrak{B}_{i}$ y $\mathfrak{A}_{i}$ son iguales, y las interpretaciones de los símbolos de $\mathcal{L}$ en ambas también son iguales. $\mathfrak{B}_{i}$ difiere de $\mathfrak{A}_{i}$ sólo en la interpretación de los nuevos simbolos de $\left.\mathcal{L}^{\prime} \backslash \mathcal{L}\right)$. Sea $D$ un filtro sobre $I$. Entonces el producto reducido $\prod_{D} \mathfrak{B}_{i}$ es una expansión del producto reducido $\prod_{D} \mathfrak{A}_{i}$. (Es decir, $\prod_{D} \mathfrak{B}_{i}$ restringida a $\mathcal{L}$ es $\prod_{D} \boldsymbol{\mathfrak { A }}_{i}$ )

Las $\Sigma_{1}^{1}$ fórmulas y los ultraproductos:

Un tipo específico de fórmulas de segundo orden son preservadas por los ultraproductos, las $\Sigma_{1}^{1}$ fórmulas, tal resultado se expresará a continuación mediante una definión y un lema, una demostración de dicho lema puede encontrarse en [[3], p. 222]:

Sea $\mathcal{L}$ un lenguaje de primer orden, y sea una expansión de $\mathcal{L}, \mathcal{L}^{\prime}=\mathcal{L} \cup$ $\left\{Q_{1}, \ldots, Q_{n}, G_{1}, \ldots, G_{m}\right\}$, donde $\operatorname{los} Q_{j}$ y los $G_{i}$ son símbolos de relación y de función, respectivamente, que no ocurren en $\mathcal{L}$. Una $\Sigma_{1}^{1}$ fórmula sobre $\mathcal{L}$ es una fórmula $\varrho$ de la siguiente forma:

$$
\left(\exists Q_{1} \ldots Q_{n} G_{1} \ldots G m\right) \phi
$$

donde $\phi$ es una fórmula (en primer orden) del lenguaje expandido $\mathcal{L}^{\prime}$. De modo que una $\Sigma_{1}^{1}$ fórmula es una fórmula de segundo orden donde todos los cuantificadores sobre relaciones y funciones ocurren al incio de dicha fórmula, y además de eso tales cuantifcadores sólo son existenciales. La definición de satisfacibilidad de una $\Sigma_{1}^{1}$ fórmula se define como sigue: $\mathrm{Si} \phi$ es una sentencia, entonces $\varrho$ ocurre en una estructura $\mathfrak{C}$ para $\mathcal{L}$ si y sólo si existe una expansión $\mathfrak{C}^{\prime}=\left(\mathfrak{C}, P_{1}, \ldots, P_{n}, F_{1}, \ldots, F_{m}\right)$ de $\mathfrak{C}$ para $\mathcal{L}^{\prime}$ tal que $\phi$ es verdad en $\mathfrak{C}^{\prime}$. Si $\phi$ tiene una variable libre $z$, entonces $\mathfrak{C}=\varrho[b]$ si y sólo si existe una expansión $\mathfrak{C}^{\prime}$ de $\mathfrak{C}$ para $\mathcal{L}^{\prime}$ tal que $\mathfrak{C}^{\prime}=\phi[b]$.

Lema 14 (Los ultraproductos también preservan las $\Sigma_{1}^{1}$ fórmulas). Sea $\mathfrak{B}$ el ultraproducto $\prod_{D} \mathfrak{A}_{i}$, donde $I$ es el conjunto de indices de los $\mathfrak{A}_{i}, f_{D}^{1}, \ldots, f_{D}^{k} \in B, y$ $\varrho\left(z_{1}, \ldots, z_{k}\right)$ es una $\Sigma_{1}^{1}$ fórmula. Entonces:

Si,

$$
\left\{i \in I: \mathfrak{A}_{i} \models \varrho\left[f^{1}(i), \ldots, f^{k}(i)\right]\right\} \in D,
$$

Entonces,

$$
\mathfrak{B} \models \varrho\left[f_{D}^{1}, \ldots, f_{D}^{k}\right] .
$$

El siguiente resultado sobre cardinales medibles se usa en el teorema que se demostrará, una prueba del mismo puede encontrarse en [[3], p. 233]:

Lema 15. Sea $\eta$ un cardinal medible, y sea $H$ un ultrafiltro sobre $\eta$, no principal $y$ $\eta$-completo. Se forma la ultrapotencia $\mathfrak{C}=\prod_{H}\langle\eta,<\rangle$. Entonces:

(i) $\mathfrak{C}$ es una estructura bien ordenada de tipo de orden mayor que $\eta$.

(ii) Para cualquier $\delta<\eta$, $d(\delta)$ es el $\delta$-ésimo elemeto de $\mathfrak{C}$. 
La demostración del siguiente teorema usa ideas de la prueba que se encuentra en el texto [3], p. 233-236]. Otra prueba de este teorema que también usa ultraproductos pero que es distinta a la que se realizará aca se puede encontrar en [[15], p. 313].

Teorema 16. Sea $\eta$ un cardinal medible. Entonces $\eta$ es un cardinal inaccesible y además $\eta$ es el $\eta$-ésimo cardinal inaccesible, es decir, existen $\eta$ cardinales inaccesibles menores que $\eta$.

Demostración. Sea $H$ un ultrafiltro no principal y $\eta$-completo sobre $\eta$. Se considera el modelo $\mathfrak{C}=\langle\eta,<, \rho\rangle_{\rho \in \eta}$ y se forma la ultrapotencia $\mathfrak{D}=\prod_{H} \mathfrak{C}$. Por el Lema anterior (15) $\mathfrak{D}$ es una estructura bien ordenada de tipo de orden mayor que $\eta$. Sea $\gamma>\eta$ el tipo de orden de $\mathfrak{D}$ y para cada $\delta<\gamma$, sea $\hat{\delta}$ el $\delta$-ésimo elemento de $\mathfrak{D}$. Como por el Corolario 9 el rango de la inmersión natural $d:\langle\eta,<, \rho\rangle_{\rho \in \eta} \longrightarrow \prod_{H} \mathfrak{D}$, rango $(d)=\{d(\delta): \delta \in \eta\}$, es un segmento inicial de $\mathfrak{D}$, entonces se cumple que $d(\delta)=\hat{\delta}$, para todo $\delta<\eta$. Por esta razón cada constante $c_{\delta}, \delta<\eta$, es interpretada por $\delta$ en la estructura $\mathfrak{C}$ y por $d(\delta)=\hat{\delta}$ en la ultrapotencia $\mathfrak{D}$.

(I) Ahora se probará que el cardinal $\eta$ es un cadinal inaccesible:

(I.1) Demostración de que $\eta$ es un cardinar regular:

(Por reducción al absurdo) Supóngase que $\eta$ no es un cardinal regular, es decir, $\eta$ es un cardinal singular. Entonces existe un ordinal $\alpha<\eta$ cofinal con $\eta$, es decir, existe una función creciente $U: \alpha \longrightarrow \eta$ cuya imagen es no acotada en $\eta$. Entonces se define la siguiente función $U^{\prime}: \eta \longrightarrow \eta$ de la siguiente manera: $U^{\prime}(\beta)=0$, para todo $\eta>\beta \leq \alpha$. Y $U^{\prime}(\beta)=U(\beta)$, para todo $\beta<\alpha$. Con esta función $U^{\prime}$ se forma el modelo $\left(\mathfrak{C}, U^{\prime}\right)$, y la ultrapotencia,

$$
\prod_{H}\left(\mathfrak{C}, U^{\prime}\right)=(\mathfrak{D}, W) .
$$

Para cada $\beta<\eta$, se tiene que:

$$
W(\hat{\beta})=W(d(\beta)) \overbrace{=}^{\infty} d\left(U^{\prime}(\beta)\right)=U^{\prime}(\beta)<\hat{\eta} .
$$

a: Como la inmersión natural $d$ es una inmersión elemental, se está aplicando la cláusula (2) de la definición de inmersión elemental.

Por lo tanto, en la ultrapotencia $(\mathfrak{D}, W)$ es verdadera la siguiente sentencia:

$$
\exists x \forall y\left(y<c_{\alpha} \rightarrow U^{\prime}(y)<x\right),(\bullet)
$$

para $x=\hat{\eta}$.

Sin embargo, como el rango de $U^{\prime}$ es cofinal en $\eta$, se cumple que la siguiente sentencia es verdad en la estructura $\left(\mathfrak{C}, U^{\prime}\right)$ :

$$
\forall x \exists y\left(y<c_{\alpha} \wedge x<U^{\prime}(y)\right),(\text { o })
$$

y por lo tanto (por la inmersión natural $d$ ) tal sentencia es también verdadera en la ultrapotencia $(\mathfrak{D}, W)$. Pero las sentencias $(\bullet)$ y (o) se contradicen mutuamente. Por lo tanto, $\eta$ no puede ser un cardinal singular, en consecuencia $\eta$ es un cardinal regular.

(I.2) Demostración de que $\eta$ es un cardinal límite fuerte:

Hay que probar que para todo cardinal $\beta<\eta$ se tiene que $2^{\beta}<\eta$. Se hará la prueba por reducción al absurdo. Supóngase que existe un cardinal $\kappa$ tal que,

$$
\kappa<\eta \leq 2^{\kappa} .
$$

En consecuencia existe un función inyectiva $W: \eta \longrightarrow P(\kappa)$. Sea $T \subseteq \eta \times \kappa$ una relación binaria, «la representante de $W »$, que se define como sigue:

$$
T(\theta, \sigma) \Longleftrightarrow \sigma \in W(\theta) .
$$

Entonces se forma la estructura $(\mathfrak{C}, T)$ y se considera la ultrapotencia: 


$$
\prod_{H}(\mathfrak{C}, T)=(\mathfrak{D}, Q)
$$

Sea $W^{\prime}$ una función de dominio $\gamma$ ( $\gamma$ es el tipo de orden de $\mathfrak{D}$ fijado anteriormente) definida de la siguiente manera:

$$
W^{\prime}(\delta)=\{\theta<\gamma: Q(\hat{\delta}, \hat{\theta})\} .
$$

Se cumple que la función $W^{\prime}$ es una función inyectiva de $\gamma$ en $P(\kappa)$, porque las dos sentencias siguientes ocurren en la estructura $(\mathfrak{C}, T)$ y por lo tanto (por la inmersión natural $d,(\mathfrak{C}, T) \preceq(\mathfrak{D}, Q))$ en la ultrapotencia $(\mathfrak{D}, Q)$ :

$$
\begin{gathered}
\forall x \forall y\left(T(x, y) \rightarrow y<c_{\kappa}\right), \\
\forall x \forall y[(x \not \equiv y) \rightarrow \exists z \neg(T(x, z) \leftrightarrow T(y, z))] .
\end{gathered}
$$

También se cumple que $W(\delta)=W^{\prime}(\delta)$, para toda $\delta<\eta$, ya que $d$ es una inmersión elemental de $(\mathfrak{C}, T)$ en $(\mathfrak{D}, Q)$. De lo anterior se puede inferir que

el conjunto $Z=W^{\prime}(\eta)$ no está en el rango de $W$, a pesar de que $Z \in P(\kappa)$. De modo que la siguiente sentencia,

$$
\exists x \forall y\left[T(x, y) \leftrightarrow \bigvee\left\{y \equiv c_{\pi}: \pi \in Z\right\}\right],
$$

es falsa en la estructura $(\mathfrak{C}, T)$, pero es verdadera en la ultrapotencia $(\mathfrak{D}, Q)$ considerando a $x=\hat{\eta}$. Esto contradice el que $d$ sea una inmersión elemental de $(\mathfrak{C}, T)$ en $(\mathfrak{D}, Q)$. Por lo tanto $\eta$ es un cardinal límite fuerte.

(II) Demostración de que $\eta$ es el $\eta$-ésimo cardinal inaccesible:

Esta parte de la demostración se realiza considerando el lema que afirma que las $\Sigma_{1}^{1}$ fórmulas son preservadas por los ultraproductos (Lema 14).

Sea $\Theta$ la clase de todos los cardinales inaccesibles, $\Delta$ la clase de todos los ordinales los cuales no son cardinales regulares, y $\Omega$ la clase de todos los ordinales los cuales no son cardinales límite fuerte. En consecuencia $\alpha \in \Theta$ si y sólo si $\alpha \notin \Delta \cup \Omega$. Se probará que $\Theta \cap \eta$ es cofinal con $\eta$. En consecuencia, dado que $\eta$ es regular, se cumple que $|\Theta \cap \eta|=\eta$, por lo tanto $\eta$ es el $\eta$-ésimo cardinal inaccesible, lo que se quiere demostrar, es decir, (II) ocurre.

Como $\eta \subseteq \Theta \cup \Delta \cup \Omega$, es suficiente con demostrar que para cada $\beta<\eta$,

(1) existe un $\theta$ tal que $\beta \leq \theta<\eta$ y $\theta \notin \Delta \cup \Omega$.

Supóngase que para algún $\beta<\eta$, (1) no ocurre. Entonces para todo $\theta<\eta$ se cumple que:

(2) $\theta<\beta \quad$ о $\theta \in \Delta$ о $\theta \in \Omega$.

Existe una $\Sigma_{1}^{1}$ fórmula $\phi_{\Delta}(v)$ tal que para cuaquier modelo $\left\langle\eta^{\prime},<\right\rangle$ donde $\eta^{\prime}$ es un ordinal, y cualquier $\tau \in \eta^{\prime}$,

$$
\text { (3) } \tau \in \Delta \text { si y sólo si }\left\langle\eta^{\prime},<\right\rangle \models \phi_{\Delta}[\tau] \text {. }
$$

$\phi_{\Delta}(v)$ se obtiene formalizando la siguiente sentencia:

« Existe un $z<v$ y existe una función $J: z \longrightarrow v$ tal que el rango de $J$ es cofinal con $\mathrm{v}$.

También existe una $\Sigma_{1}^{1}$ fórmula $\varphi_{\Omega}(v)$ tal que para cuaquier modelo $\left\langle\eta^{\prime},<\right\rangle$ donde $\eta^{\prime}$ es un ordinal, y cualquier $\tau \in \eta^{\prime}$,

$$
\text { (4) } \tau \in \Omega \text { si y sólo si }\left\langle\eta^{\prime},<\right\rangle \models \varphi_{\Omega}[\tau] \text {. }
$$

$\phi_{\Delta}(v)$ se obtiene formalizando la siguiente sentencia:

« Existe un $z<v$ y existe una relación $T \subseteq v \times z$ tal que $T$ representa a una función inyectiva de $v$ en $P(z)$ ».

Anteriormente (en esta demostración) se ha explicado como definir una relación $T$ que representa a una función inyectiva $W: v \longrightarrow P(z)$ y también se ha explicado como decir que $W$ es inyectiva usando a $T$. 
A partir de (2), (3) y (4) se tiene que la fórmula,

$$
\text { (5) } v<c_{\beta} \vee \phi_{\Delta}(v) \vee \varphi_{\Omega}(v) \text {, }
$$

es verdadera en $\langle\eta,<\rangle$ para todo $v \in \eta$.

Moviendo los cuantificadores de segundo orden para el inicio se puede apreciar que (5) es equivalente a una $\Sigma_{1}^{1}$ fórmula. Por lo tanto, por el Lema 14 (Las fórmulas $\Sigma_{1}^{1}$ son preservadas por ultraproductos), para cualquier $g_{H} \in B$ la fórmula (5) es satisfecha por $g_{H}$ en $\langle D,<\rangle$, donde $\langle D,<\rangle$ es la ultrapotencia correspondiente a la estructura $\langle\eta,<\rangle$, construida con el ultrafiltro $H$ no principal y $\eta$-completo sobre $\eta$. Dado que $\langle D,<\rangle$ es isomorfa a $\langle\gamma,<\rangle$, la fórmula (5) es verdad en $\langle\gamma,<\rangle$, para toda $v \in \gamma$. Poniendo $v=\eta$ se tiene que:

\section{$\eta<\beta \quad$ o $\phi_{\Delta}(\eta)$ о $\varphi_{\Omega}(\eta)$.}

Usando (3) y (4), con $\gamma=\eta^{\prime}$, se tiene que:

$\eta<\beta$ о $\quad \eta \in \Delta$ o $\eta \in \Omega$. Pero esto contradice la hipótesis de que $\beta<\eta$ y que $\eta$ es un cardinal inaccesible. Con esto termina la demostración del Teorema.

\section{Tercer teorema sobre CARdinales medibles: El teorema de Scott.}

El tercer teorema sobre cardinales medibles que se demostrará (usando ultraproductos) es el teorema de Scott 22]: Si existen cardinales medibles, entonces el axioma de constructibilidad $(V=L)$ es falso.

Es conocido que el axioma de constructibilidad implica la «hipótesis generalizada del continuo» $\left(\forall \alpha \in \operatorname{Ord}\left(2^{\aleph_{\alpha}}=\aleph_{\alpha+1}\right)\right)$ y al axioma de elección (ver $\left.[11,13,17]\right)$.

También es conocido que Gödel demostró que se cumple $\langle\mathbf{L}, \in\rangle=\mathbf{V}=\mathbf{L}$ (ver $[11,13,17])$, entonces el teorema de Scott implica que en $\mathbf{L}$ no existen cardinales medibles.

La prueba del teorema de Scott se realiza utilizando ideas principalmente de Chang y Keisler en [3, p. 238-239]. Como en la demostración de los dos teoremas anteriores sobre cardinales medibles también se usan en esta prueba lenguajes infinitarios (los cuales son preservados por los ultraproductos). Otra demostración de este teorema que también usa ultraproductos pero que es distinta a la que se realizará aca se puede encontrar en [[15], p. 311].

A continuación se enuncian algunos resultados y definiciones previas:

$\mathbf{V}$ es la clase de los conjuntos bien fundamentados («La jerarquía acumulativa de conjuntos de Zermelo») que se define usando inducción transfinta en la clase de los ordinales:

$$
\begin{gathered}
V_{0}=\emptyset \\
V_{\alpha+1}=P\left(V_{\alpha}\right) \\
V_{\lambda}=\bigcup_{\beta<\lambda} V_{\beta}, \lambda \text { límite. } \\
\mathbf{V}=\bigcup_{\alpha \in O r d} V_{\alpha} .
\end{gathered}
$$

Si $x \in \mathbf{V}$ entonces el rango de $x, \rho(x)$, es el menor ordinal $\alpha$ tal que $x \in V_{\alpha+1}$.

Para cada $\alpha \in$ Ord ocurre que $V_{\alpha}$ es un conjunto transitivo ( $X$ es transitivo si $\forall z(z \in X \rightarrow z \subseteq X)$. También se cumple que: (i) Para cada $\alpha, \beta \in$ Ord: Si $\alpha \leq \beta$, entonces $V_{\alpha} \subseteq V_{\beta}$. (ii) Para cada ordinal $\alpha$ : $V_{\alpha} \cap$ Ord $=\alpha$. Y (iii) si $\eta$ es un cardinal medible, entonces $\left\langle V_{\eta}, \in\right\rangle$ es un modelo de $Z F C$. (ver $[3,13,15]$ ).

$\mathbf{L}$ es la clase de los conjuntos constructibles de Gödel que se define (informalmente) usando inducción transfinita en la clase de los ordinales:

Antes de dar la definición se introduce el concepto de definibilidad en una estructura (ver $[3,5])$ : Consideremos una estructura $\mathfrak{A}=\left\langle A,<R_{\beta}^{\mathfrak{A}}>_{\beta \in \gamma},<f_{\mu}^{\mathfrak{A}}>_{\mu \in \delta},<c_{\xi}^{\mathfrak{A}}>_{\xi \in \eta}\right\rangle$ para un lenguaje $\mathcal{L}$. Se dice que un subconjunto $B \subseteq A$ es definible en $\mathfrak{A}$ si existe una 
fórmula $\varphi(x)$ del lenguaje $\mathcal{L}$ tal que $B=\{z \in A: \mathfrak{A} \models \varphi[z]\}$. Se dice que $B$ es definible en $\mathfrak{A}$ con parámetros si existe fórmula $\varphi\left(x, x_{1}, \ldots, x_{n}\right)$ del lenguaje $\mathcal{L}$ y existen $a_{1}, \ldots, a_{n} \in A$ tal que: $B=\left\{z \in A: \mathfrak{A}=\varphi\left[z, a_{1}, \ldots, a_{n}\right]\right\}$.

$$
\begin{gathered}
L_{0}=\emptyset \\
L_{\alpha+1}=\left\{X \subseteq L_{\alpha}: X\right. \text { es definible en la estructura } \\
\left.\left\langle L_{\alpha}, \in,\left\langle b: b \in L_{\alpha}\right\rangle\right\rangle\right\} \\
L_{\lambda}=\bigcup_{\beta \in \lambda} L_{\beta}, \lambda \text { límite. } \\
\mathbf{L}=\bigcup_{\alpha \in \text { Ord }} L_{\alpha}
\end{gathered}
$$

Es claro que en el paso sucesor la expresión $« X$ es definible en la estructura $\left\langle L_{\alpha}, \in\right.$ , $\left.\left\langle b: b \in L_{\alpha}\right\rangle\right\rangle_{\gg}$ supone que se tiene un lenguaje de primer orden con identidad, cuyos símbolos no lógicos son: Una constante $\underline{b}$ para cada $b \in L_{\alpha}$ y un símbolo relacional binario $\in$ para la relación de pertenencia $\in$. Sin embargo también se puede hablar de «definible con parámetros» y eliminar las nuevas constantes agregadas al lenguaje inicial. Formalizaciones en ZF de la definición intuitiva de $\mathbf{L}$ pueden encontrarse en $[13,17]$.

Se cumple que para $\alpha \in$ Ord: $L_{\alpha}$ es transitivo y $L_{\alpha} \subseteq V_{\alpha}$. También ocurre que para cada $n \in \aleph_{0}\left(L_{n}=V_{n}\right)$. Y también se cumple que: (i) $L_{\omega}=V_{\omega}$. Y (ii) para cada ordinal $\alpha: L_{\alpha} \cap$ Ord $=\alpha$.

Sea $\alpha$ un cardinal infinito. $H(\alpha)$ es el conjunto de todos los conjuntos hereditariamente de cardinal menor que $\alpha$, es decir, $H(\alpha)=\{x:|T C(x)|<\alpha\}$, donde dado un conjunto $D, T C(D)$ es la clausura transitiva de $D$, es decir, $T C(D)$ es el menor conjunto transitivo (con respecto a la relación inclusión) que contiene a $D$. Se cumple que $z \in H(\alpha)$ si y sólo si existe un conjunto transitivo $w$ tal que $z \subseteq w$ y $|w|<\alpha$. Entre las propiedades $H(\alpha)$ se encuentran las siguientes (ver [[3], p. 237] y [[17], p. 130-133]):

Lema 17. 1. $H(\alpha)$ es un subconjunto transitivo de $V_{\alpha}$.

2. Si $\alpha \leq \beta$, entonces $H(\alpha) \subseteq H(\beta)$.

3. $\alpha \subseteq H(\alpha)$.

4. Si $\alpha>\aleph_{0}$ es un cardinal regular, entonces $\langle H(\alpha), \in\rangle$ es un modelo de $Z F-P$.

5. $H(\alpha) \cap \operatorname{Ord}=\alpha$.

Como se definió anteriormente en este artículo una relación binaria $E$ es bien fundamentada si no existen secuencias infinitas decrecientes con respecto a $E$, es decir, si no existen secuencias $\left\{x_{n}: n \in \aleph_{0}\right\}$ tal que: ..., $x_{4} E x_{3}, x_{3} E x_{2}, x_{2} E x_{1}, x_{1} E x_{0}$.

Sea $X$ un conjunto y $E$ una relación binaria bien fundamentada sobre $X$. La estructura $\langle X, E\rangle$ se llama estructura o modelo bien fundamentado.

Sea $\langle X, E\rangle$ una estructura bien fundamentada. Se dice que $a \in X$ es un ordinal de $\langle X, E\rangle$ si y sólo si ocurre lo siguiente:

$$
\begin{gathered}
\langle X, E\rangle \models \forall x \forall y[(x \in a \wedge y \in a \rightarrow x \in y \vee y \in x \vee x \equiv y) \wedge \\
(x \in a \wedge y \in x \rightarrow y \in a)] .
\end{gathered}
$$

Hecho $\star$ : Dada la definición anterior se cumple que $u$ es un ordinal de $\langle H(\alpha), \in\rangle$ si y sólo si $u \in \alpha$. (ver [[3, p. 238]).

El siguiente resultado afirma que las relaciones bien fundamentadas sobre un conjunto dado $X$ bien ordenan los ordinales de dicho conjunto $X$ si $(X, E) \models Z F-P$, una prueba del mismo puede encontrarse en [[3], p. 238]:

Lema 18. Sea $\langle X, E\rangle$ un modelo bien fundamentado de ZF-P. Entonces el conjunto de los ordinales de $\langle X, E\rangle$ está bien ordenado por $E$. 
Sea $\langle X, E\rangle$ un modelo bien fundamentado de $Z F-P$. El tipo de orden de $\langle X, E\rangle$ es el tipo de orden del conjunto de ordinales de $\langle X, E\rangle$ según $E$.

Formulación del axioma de constructibilidad (la versión de dicho axioma que se utilizará en esta demostración):

Axioma de constructibilidad: Para cualquier cardinal regular $\mu>\aleph_{0}$ (todo modelo bien fundamentado $\langle A, S\rangle$ de $Z F-P$ de tipo de orden $\mu$ es isomorfo a $\langle H(\mu), \in\rangle)$.

Observación: Se puede apreciar que la versión del axioma de constructibilidad que se acaba de formular es diferente a la que se usa normalmente en teoría de conjuntos: $\mathbf{V}=\mathbf{L}$, expresión que denota a la proposición $\forall x \exists \alpha \in \operatorname{Ord}\left(x \in L_{\alpha}\right)$. La razón de la elección es que la versión del axioma de constructibilidad que utilizaremos conviene más que la versión $\mathbf{V}=\mathbf{L}$ a los fines de poder aplicar las técnicas que usaremos en la demostración del teorema de Scott. Como se dijo anteriormente existen pruebas distintas del teorema de Scott de la que haremos en este artículo las cuales usan la versión $\mathbf{V}=\mathbf{L}$ del axioma de constructibilidad, por ejemplo en $[6,13,15,16]$, sin embargo, se puede demostrar en $Z F C$ que la versión del axioma de constructibilidad que utilizaremos es equivalente a la versión $\mathbf{V}=\mathbf{L}$. No daremos una demostración rigurosa de tal equivalencia pues dispersaría al lector de la idea principal del artículo ya que dicha demostración requiere de la introducción de conceptos y resultados adicionales (que no están en el artículo), no obstante, mencionaremos algunas ideas fundamentales que se pueden aplicar en la demostración de dicha equivalencia y ofreceremos un esbozo de una prueba que es original del autor de este artículo: Primero que nada es conveniente decir, siguiendo a [3], que dicha versión afirma intuitivamente que $H(\mu)$ es muy estrecho ¿y qué significa esto? se puede explicar muy bien con la siguiente proposición que también es una versión equivalente del axioma de constructibilidad, la cual llamaremos «axioma de constructibilidad (versión 3)»: Para cualquier cardinal regular $\mu>\aleph_{0}$ (no existe un subconjunto propio $M \subset H(\mu)$ tal que $M$ es transitivo, $\alpha \subseteq M y\langle M, \in\rangle$ es un modelo de $Z F-P)$. Con respecto a $H(\mu)$ es bueno tener presente la cláusula (4) del lema anterior 17, cuya prueba se puede encontrar en los textos mencionados cuando se enunció el lema. Ahora bien, una prueba de las equivalencias mencionadas se puede hacer usando los siguientes resultados (y las ideas contenidas en el mismos): (a) Para cualquier cardinal regular $\kappa>\aleph_{0}: L_{\kappa} \mid=Z F-P$. Una prueba de dicho resultado (a) puede encontrarse en [20], p.84]. Y (b) Si $\mathbf{V}=\mathbf{L}$, entonces para todo $\kappa, H(\kappa)=L_{\kappa}$. Una prueba de tal resultado (b) se puede encontrar en [[20, p. 84-85]. Entre las ideas y resultados contenidos en las demostraciones de (a) y (b) estan: (1) La relación funcional de los constructibles $\alpha \mapsto L_{\alpha}$ es absoluta para modelos transitivos de $Z F-P,(2)$ absoluticidad en general, (3) teorema de reflexión, (4) teorema de Löwenheim-Skolem, (5) teorema de colapso transitivo de Mostowski y (6) lema de condensación. Finalmente, para probar que las tres versiones del axioma de constructibilidad son equivalentes, usando las ideas y los resultados anteriores (entre otros), se puede proceder así: Primero se prueba que: (I) axioma de construcibilidad $\Rightarrow$ axioma de constructibilidad (versión 3). Luego se demuestra que: (II) axioma de constructibilidad (versión 3) $\Rightarrow \mathbf{V}=\mathbf{L}$. Y por último se demuestra que: (III) $\mathbf{V}=\mathbf{L} \Rightarrow$ axioma de constructibilidad. Esbozo de la prueba de (I): Sea $\mu$ un cardinal regular tal que $\mu>\aleph_{0}$. Y supongamos que existe un $M \subset H(\mu)$ tal que $M$ es transitivo, $\alpha \subseteq M$ y $\langle M, \in\rangle$ es un modelo de $Z F-P$. Entonces, por la hipótesis, $\langle M, \in\rangle$ es isomorfo a $\langle H(\mu), \in\rangle$. Sea $\pi$ la función biyectiva de $M$ en $H(\mu)$ que establece el isomorfismo. En consecuencia $\pi$ es la función identidad, es decir, $\pi(x)=x$ $(\forall x \in M)$, y por lo tanto, $M=H(\mu)$, pues dos clases transitivas distintas no pueden ser $\in$-isomorfas (ver teorema 26 del texto [15], p. 74]). Esbozo de la prueba de (II): Sea $x \in \mathbf{V}$. Consideramos la clausura transitiva del conjunto unitario $\{x\}$, es decir, $T C(\{x\})$. Sea $\alpha$ el cardinal de $T C(\{x\})$ y $\alpha^{+}$es cardinal sucesor de $\alpha$. Ocurre que 
$x \in H\left(\alpha^{+}\right)$. Consideramos $\left\langle H\left(\alpha^{+}\right), \in\right\rangle$. Por el Lema 17 tenemos que $\left\langle H\left(\alpha^{+}\right), \in\right\rangle$ es un modelo de $Z F-P$. Por otro lado tenemos que, por la absoluticidad de $\alpha \mapsto L_{\alpha}$, $L_{\alpha^{+}} \subseteq H\left(\alpha^{+}\right)$. También ocurre que $\left\langle L_{\alpha^{+}}, \in\right\rangle$ es un modelo de $Z F-P$ (resultado (a) anteriormente referido). Entonces $x \in L_{\alpha^{+}}$, pues si esto no ocurriera se contradice la hipótesis. Por lo tanto, $x \in \mathbf{L}$. Esbozo de la prueba de (III): Sea $\mu$ un cadinal regular tal que $\mu>\aleph_{0}$. Y sea $\langle A, S\rangle$ un modelo bien fundamentado de $Z F-P$ de tipo de orden $\mu$. Entonces por el teorema del colapso transitivo de Mostowski se tiene que existe una clase transitiva $M$ tal que $\langle A, S\rangle$ y $\langle M, \in\rangle$ son isomorfas. Como $\alpha \mapsto L_{\alpha}$ es absoluta, se tiene que $L_{\mu} \subseteq M$. Sea $x \in M$. Entonces, como por hipótesis $\mathbf{V}=\mathbf{L}$, $x \in \mathbf{L}$. Y como $\rho(x)<\mu$ y $L_{\mu}=\{z \in \mathbf{L}: \rho(\mathbf{z})<\mu\}$, se tiene que $x \in L_{\mu}$. De modo que $M \subseteq L_{\mu}$. En consecuencia, $M=L_{\mu}$. Por otro lado, por el resultado (b) referido anteriormente, ocurre que $M=L_{\mu}=H(\mu)$. Luego, $\langle A, S\rangle$ y $\langle H(\mu), \in\rangle$ son isomorfas. Fin del esbozo de la prueba (III), y con esto también termina el esbozo de la prueba de las equivalencias de las tres versiones mencionadas del Axioma de constructibilidad.

Teorema 19 (Teorema de Scott). Si existe un cardinal medible, entonces el axioma de constructibilidad es falso. (En consecuencia el axioma de constructibilidad implica que no existen cardinales medibles).

Demostración. Sea $\eta$ el primer cardinal medible. Y sea $\theta=\left|2^{2^{2^{\eta}}}\right|^{+}$. Entonces $V_{\eta+3}$ es un conjunto transitivo de cardinalidad menor que $\theta$, pues:

$$
\begin{gathered}
\left.\left|V_{\eta+1}\right|=\left|P\left(V_{\eta}\right)\right|=2^{\left|V_{\eta}\right|}\left(\eta \text { es inaccesible, }\left|V_{\eta}\right|=\eta\right)=2^{\eta}, \quad(\text { ver }[15], \text { p. } 72]\right) \\
\left|V_{\eta+2}\right|=\left|P\left(V_{\eta}+1\right)\right|=2^{\left|V_{\eta+1}\right|}=2^{2^{\eta}} \\
\left|V_{\eta+3}\right|=\left|P\left(V_{\eta+2}\right)\right|=2^{\left|V_{\eta+2}\right|}=2^{2^{2^{\eta}}}<\theta .
\end{gathered}
$$

Y también $\theta$ es un cardinal regular porque todo cardinal sucesor es regular (ver [ [5], p. 92]).

En consecuencia $V_{\eta+3} \in H(\theta)$ y $\langle H(\theta), \in\rangle$ es un modelo de $Z F-P$, porque $\theta$ es un cardinal regular no numerable (Lema 17 , cláusula 4$)$.

Sea $H$ un ultrafiltro no principal y $\eta$-completo sobre $\eta$. Con $H$ y $\langle H(\theta), \in\rangle$ se forma la ultrapotencia,

$$
(\mathfrak{D}, R)=\prod_{H}\langle H(\theta), \in\rangle .
$$

Entonces como el concepto «relación bien fundamentada» se puede caracterizar con una fórmula del lenguaje infinitario $\mathcal{L}_{\aleph_{1} \aleph_{1}}$,

$$
\mathrm{RBF}:\left(\forall x_{0} x_{1} x_{2} \ldots\right) \neg \bigwedge\left\{P\left(x_{n+1}, x_{n}\right): n \in \aleph_{0}\right\},
$$

y los ultraproductos preservan las formulas infinitarias (Lema 10), entonces $(\mathfrak{D}, R)$ es un modelo bien fundamentado de $Z F-P$ (los axiomas de $Z F-P$ se cumplen en $(\mathfrak{D}, R)$ porque existe la inmersión (elemental) natural $d$ de $\langle H(\theta), \in\rangle$ en $(\mathfrak{D}, R))$. En consecuencia, por el Lema 18 , el conjunto de los ordinales de $(\mathfrak{D}, R)$ está bien ordenado por $R$.

Proposición: El conjunto de los ordinales de $(\mathfrak{D}, R)$ tiene tipo de orden $\theta$.

Prueba de la proposición: La inmersión natural $d$ es un isomorfismo de $\langle H(\theta), \in\rangle$ en $(\mathfrak{D}, R) \uparrow d(H(\theta))$. En consecuencia el conjunto de los ordinales de $(\mathfrak{D}, R)$ tiene tipo de orden al menos $\theta$ (tomar en cuenta el Hecho $\star$ ). Sea $z$ cualquier ordinal de $(\mathfrak{D}, R)$. Entonces se concluye que $z=g_{H}$, para alguna función $g: \eta \longrightarrow \theta$.

(Nota: Usando la definición de «ordinal de una estructura bien fundamentada» y el Lema 18 de buen orden se puede probar que todo ordinal z de la ultrapontencia $(\mathfrak{D}, R)=\prod_{H}\langle H(\theta), \in\rangle$ tiene la forma $g_{H}$, para alguna función $g: \eta \longrightarrow \theta$.) 
Como $\operatorname{cof}(\theta)>\eta$, entonces $g$ no es cofinal en $\theta$ es decir, el rango de $g$ está acotado en $\theta$. Por lo tanto existe un $\delta<\theta$ tal que $g: \eta \longrightarrow \delta$, es decir, $g \in{ }^{\eta} \delta$. De lo anterior se sigue que si $w R z$, entonces $w=j_{H}$ para alguna $j \in{ }^{\eta} \delta$. En consecuencia el conjunto $|\{w: w R z\}| \leq \delta^{\eta}$, y como $\delta \leq 2^{2^{2^{\eta}}}$ (por definición de cardinal sucesor (ver [[5], p. $76])$ ), entonces $\delta^{\eta}<\theta$ : En efecto, como $\delta \leq 2^{2^{2^{\eta}}}$, entonces por propiedades de potencias de cardinales (ver [[13, p. 29]) se tiene que $(\delta)^{\eta} \leq\left(2^{2^{2^{\eta}}}\right)^{\eta}=2^{\left(2^{2^{\eta}}\right) \cdot \eta}=2^{\left(2^{2^{\eta}}\right)}<\theta$. Esto muestra que cualquier ordinal de $(\mathfrak{D}, R)$ tiene menos de $\theta$ predecesores. En consecuencia el conjunto de los ordinales de $(\mathfrak{D}, R)$ tiene a lo sumo tipo de orden $\theta$. Por lo tanto, el tipo de orden del conjunto de los ordinales de $(\mathfrak{D}, R)$ es $\theta$. Ha culminado la prueba de la proposición.

Sea $\phi(z)$ una fórmula de la teoría de conjuntos que afirma «z es el primer cardinal medible». Cuando se escribe en detalle dicha fórmula se puede apreciar que sus cuantificadores pueden ser restringidos a $P(P(P(z))$ ) (ver [[3], p. 239]). En consecuencia, en el modelo $\langle H(\theta), \in\rangle$ un elemento $a$ satisface $\phi(z)$ si y solamente si $a$ es realmente el primer cardinal medible, $a=\eta$. De modo que en la ultrapotencia $(\mathfrak{D}, R)$ el único elemento que satisface $\phi(z)$ es $d(\eta)$. El ordinal $d(\eta)$ es mayor que el $\eta$-ésimo ordinal de $(\mathfrak{D}, R)$ ¿por qué? por el Lema 15 y considerando la estructura $\langle\eta,<\rangle$ se tiene que la ultrapotencia que se forma con la misma usando el ultrafiltro $H$ no principal y $\eta$-completo sobre $\eta,(\mathfrak{C}, K)=\prod_{H}\langle\eta, \in\rangle$, es una estructura bien ordenada de tipo de orden mayor que $\eta$ y para cualquier $\delta<\eta, d(\delta)$ es el $\delta$-ésimo elemento de $\prod_{H}\langle\eta, \in\rangle$. Se cumple que $\prod_{H}\langle\eta, \in\rangle$ está inmersa en la ultrapotencia $(\mathfrak{D}, R)=\prod_{H}\langle H(\theta), \in\rangle$ ¿cuál es una inmersión? La siguiente: Para cada $f \in{ }^{\eta} \eta$ se considera $f_{H} \in(\mathfrak{C}, K)=\prod_{H}\langle\eta, \in\rangle$ y $f_{H} \in(\mathfrak{D}, R)=\prod_{H}\langle H(\theta), \in\rangle$, dicha correspondencia es una inmersión. Como tal inmersión preserva el orden y la ultrapontencia $(\mathfrak{C}, K)$ esta bien ordenda y su tipo de orden es mayor que $\eta$, entonces $(\mathfrak{C}, K)$ es un segmento inicial de los ordinales de la ultrapotencia bien fundamentada $(\mathfrak{D}, R)$ (todo ordinal $z$ de las ultrapotencias $(\mathfrak{D}, R)=\prod_{H}\langle H(\theta), \in\rangle$ o $(\mathfrak{C}, K)=\prod_{H}\langle\eta, \in\rangle$ tiene la forma $g_{H}$, para alguna función $g: \eta \longrightarrow \delta)$. Por lo tanto existe una función $u \in \in^{\eta} \eta$ tal que la clase de equivalencia $u_{H}$ es el $\eta$-ésimo ordinal de $(\mathfrak{C}, R)$ y de $(\mathfrak{D}, R)$, es decir, $u_{H}$ tiene $\eta$ predecesores. Considerando a $d(\eta)$ en la ultrapotencia $(\mathfrak{D}, R)$ se tiene que ella es la clase de equivalencia de la función constante en $\eta:\langle\eta: \delta \in \eta\rangle$. Si se llama a tal función constante $c$ se puede reescribir la misma así : $c: \eta \longrightarrow H(\theta)$ tal que para cada $\delta \in \eta(c(\delta)=\eta)$. Por otro lado, $u_{H}$ en $(\mathfrak{D}, R)$ es la clase de equivalencia de la función $u \in{ }^{\eta} \eta$. Es decir, para cada $\delta \in \eta(u(\delta) \in c(\delta)=\eta))$. Por lo tanto, $\eta=\{\delta: u(\delta) \in c(\delta)=\eta\} \in H$. Entonces, por la definición de ultrapotencia, se cumple que: $u_{H} R d(\eta)$. En consecuencia las estructuras $\langle H(\theta), \in\rangle$ y $(\mathfrak{D}, R)$ no son isomorfas, pues cualquier isomorfismo asigna $\eta$ al $\eta$-ésimo ordinal de $(\mathfrak{D}, R), \hat{\eta}$, y $\hat{\eta}$ no satisface $\phi(z)$. Por lo tanto, el axioma de constructibilidad es falso. Con esto termina la demostración del Teorema.

Problemas abiertos: Vale la pena destacar que algunos problemas abiertos de teoría de conjuntos y/o teoría de modelos relacionados con cardinales medibles, cardinales grandes y ultraproductos pueden encontrarse en los textos [16] y [ [3], p. 597-602 (Apéndice B)].

\section{REFERENCIAS}

[1] Bell, J., Infinitary Logic, Enciclopedia de Filosofía de la universidad de Stanford, 2016, https://plato.stanford.edu/entries/logic-infinitary/

[2] Corbillón, M., Análisis real no estándar, Tesis de licenciatura en Matemáticas, Tutor: Dr. Josep Maria Font Llovet, Facultat de Matemátiques, Universitat de Barcelona, 2015.

[3] Chang, C., Keisler, H., Model Theory, Dover Publications, 2012.

[4] Di Prisco, C., Introdución a la Lógica Matemática, Emalca Amazonia, 2009.

[5] Di Prisco, C., Teoría de Conjuntos, Universidad Central de Venezuela: Consejo de Desarrollo Científico y Humanístico, 2009.

[6] Di Prisco, C., Inmersiones elementales y grandes cardinales, Notas no publicadas, 1982.

[7] Enderton, H., Una Introducción Matemática a la Lógica, Universidad Nacional Autónoma de México, 2004. 
[8] Enderton, H., Elements of Set Theory, Academic Press, 1977.

[9] Ebbingahaus, H., Flum, J., Thomas, W., Mathematical Logic, Springer, 1996.

[10] Galindo, F., Una presentación de la demostración directa del teorema de compacidad de la lógica de primer orden que usa el método de ultraproductos, Una Investig@ción, Vol. VIII, $N^{\circ}$ 15 (2016).

[11] Gödel, K., Obras completas, Alianza, 1981.

[12] Hrbacek, K., Jech, T., Introducton to set theory, Marcel Dekker, 1999.

[13] Jech, T., Set Theory, Springer, 2000.

[14] Jech, T., The Axiom of Choice, Dover Publications, 2008.

[15] Jech, T., Set Theory, Academic Press, 1978.

[16] Kanamori, A., The Higher Infinite. Large Cardinal in Set Theory from their Beginnings, Springer, 1997.

[17] Kunen, K., Set Theory. An Introduction to Independence Proofs, College Publications, 2011.

[18] Loś, J., Quelques remarques, théorèmes et problèmes sur les classes définissables d'algèbres, en «Mathematical Interpretation of Formal Systems» (T. Skolem et al.,eds.), North-Holland Publishing Co., 1955, 98-113.

[19] Manzano, M., Teoría de Modelos, Alianza, 1989.

[20] Marks, A., Set Theory, Notas, Mathematics UCLA, 2020. (Estan en la web).

[21] Mendelson, E., Introduction to Mathematical Logic, Chapman and Hall/CRL, 2009.

[22] Scott, D., Measurable cardinals and constructive sets, Bull.Acad. Polon. Sci. Sér. Sci. Math. Astrom. Phys. 9, 521-524, 1961

Dirección del autor:

Universidad Central de Venezuela,

Facultad de Humanides y Educación,

Escuela de Filosofía,

Departamento de Lógica y Filosofía de la Ciencia.

Venezuela.

Colaborador Visitante del Departamento de Matemáticas del Instituto Venezolano de Investigaciones Científicas.

Venezuela.

e-mail: franklin.galindo@ucv.ve 